\title{
NONUNIFORM TRANSFORMATION FIELD ANALYSIS APPLIED TO DISCRETE ELASTOPLASTIC MICROSTRUCTURE
}

\author{
K. Mikeš ${ }^{*}$, M. Jirásek ${ }^{* *}$, J. Zeman ${ }^{* * *}$
}

\begin{abstract}
In this contribution, we focus on numerical simulation of discrete elastoplastic microstructures with periodic boundary conditions. The idea of nonuniform transformation field analysis, originally proposed by Michel and Suquet, is used for simplification of the given problem by reducing the number of internal variables represented by plastic strains. For that purpose, the plastic strains are approximated by linear combinations of selected plastic modes. Two approaches to the selection of plastic modes are proposed and compared using a simple two-dimensional example. Their accuracy is evaluated by comparing the results with the exact solution.
\end{abstract}

Keywords: Transformation Field Analysis, Model reduction, Plasticity, Microstructure.

\section{Introduction}

This work is focused on numerical simulations of random microstructures with periodic boundary conditions. Numerical simulation of periodic cells plays an important role in numerical homogenization. Appropriate simplification of this problem that can provide a fast macroscopic response with an acceptable error can be very useful, especially in cases when one simulation of similar microstructures with different settings is realized multiple times, e.g., in optimization or parameter identification. For that purpose, the central idea of nonuniform transformation field analysis is used in this work. A given problem is simplified by approximation of internal variables represented by plastic micro-level strains.

\section{Full model}

\subsection{Kinematics}

The considered microstructure is represented by nodes connected by elastoplastic links. The kinematics of a periodic unit cell can be described by displacements of particles located "inside" the periodic cell, denoted by $d_{m}$. Furthermore, displacements of ghost particles, denoted by $d_{g}$, are employed to enforce periodicity of the unit cell; see Fig. 1 (a). The macroscopic strain of the unit cell is denoted by e and the micro-level strain is described by the elongations of the links, denoted by $\mathbf{e}_{\mathrm{m}}$.

The displacements of ghost particles can be expressed in terms of the displacements of their periodic images and of the macroscopic strain

$$
\mathbf{d}_{\mathrm{g}}=\mathbf{I}_{\mathrm{m}} \mathbf{d}_{\mathrm{m}}+\mathbf{P}_{\mathrm{e}} \mathbf{e}
$$

where $\mathbf{I}_{\mathrm{m}}$ is a Boolean matrix which assigns to each ghost particle its "master particle" and $\mathbf{P}_{\mathrm{e}}$ reflects the influence of macroscopic strains on the relative displacements between masters and their ghost images.

The link elongations can be expressed as

$$
\mathbf{e}_{\mathrm{m}}=\mathbf{B}_{\mathrm{m}}^{*} \mathbf{d}_{\mathrm{m}}+\mathbf{B}_{\mathrm{g}}^{*} \mathbf{d}_{\mathrm{g}}
$$

Ing. Karel Mikeš: Faculty of Civil Engineering, Czech Technical University in Prague, Thákurova 7; 166 29 , Prague; CZ, Mikes.Karel@cvut.cz

** Prof. Ing. Milan Jirásek, DrSc.: Faculty of Civil Engineering, Czech Technical University in Prague, Thákurova 7; 16629 , Prague; CZ, Milan.Jirasek@fsv.cvut.cz

*** Prof. Ing. Jan Zeman, Ph.D.: Faculty of Civil Engineering, Czech Technical University in Prague, Thákurova 7; 166 29, Prague; CZ, Jan.Zeman@cvut.cz 
where $\mathbf{B}_{\mathrm{m}}^{*}$ and $\mathbf{B}_{\mathrm{g}}^{*}$ are parts of the geometric matrix describing the arrangement of particles and links inside the periodic cell.

The ghost degrees of freedom can be eliminated by substituting (1) into (2) and the micro-strains can be written in terms of the independent micro-displacements and macro-strains as

$$
\mathbf{e}_{\mathrm{m}}=\mathbf{B}_{\mathrm{m}}^{*} \mathbf{d}_{\mathrm{m}}+\mathbf{B}_{\mathrm{g}}^{*}\left(\mathbf{I}_{\mathrm{m}} \mathbf{d}_{\mathrm{m}}+\mathbf{P}_{\mathrm{e}} \mathbf{e}\right)=\mathbf{B}_{\mathrm{m}} \mathbf{d}_{\mathrm{m}}+\mathbf{B}_{\mathrm{e}} \mathbf{e}=\left[\mathbf{B}_{\mathrm{m}}, \mathbf{B}_{\mathrm{e}}\right]\left\{\begin{array}{c}
\mathbf{d}_{\mathrm{m}} \\
\mathbf{e}
\end{array}\right\}
$$

where

$$
\begin{aligned}
\mathbf{B}_{\mathrm{m}} & =\mathbf{B}_{\mathrm{m}}^{*}+\mathbf{B}_{\mathrm{g}}^{*} \mathbf{I}_{\mathrm{m}} \\
\mathbf{B}_{\mathrm{e}} & =\mathbf{B}_{\mathrm{g}}^{*} \mathbf{P}_{\mathrm{e}}
\end{aligned}
$$

\subsection{Stored energy}

For all links in the periodic cell, we consider linear elasticity and linear hardening described by elastic modulus $E$ and plastic modulus $H$. Then the stored energy can be expressed as

$$
E_{\text {int }}^{(\mathrm{ep})}\left(\mathbf{e}_{\mathrm{m}}, \mathbf{p}_{\mathrm{m}}\right)=\frac{1}{2}\left(\mathbf{e}_{\mathrm{m}}-\mathbf{p}_{\mathrm{m}}\right)^{T} \mathbf{D}_{\mathrm{m}}\left(\mathbf{e}_{\mathrm{m}}-\mathbf{p}_{\mathrm{m}}\right)+\frac{1}{2} \mathbf{p}_{\mathrm{m}}^{T} \mathbf{H}_{\mathrm{m}} \mathbf{p}_{\mathrm{m}}
$$

where $\mathbf{H}_{\mathrm{m}}$ and $\mathbf{D}_{\mathrm{m}}$ are diagonal matrices describing the hardening stiffness $(H A / L)$ and elastic stiffness $(E A / L)$, respectively. $A$ denotes the sectional area of the link and $L$ is its length.

The derivative of the stored energy with respect to the micro-strains gives the micro-stresses

$$
\mathbf{s}_{\mathrm{m}}=\frac{\partial E_{\mathrm{int}}^{(\mathrm{ep})}\left(\mathbf{e}_{\mathrm{m}}, \mathbf{p}_{\mathrm{m}}\right)}{\partial \mathbf{e}_{\mathrm{m}}}=\mathbf{D}_{\mathrm{m}}\left(\mathbf{e}_{\mathrm{m}}-\mathbf{p}_{\mathrm{m}}\right)
$$

For given macroscopic strains e, the particles will find positions that minimize the stored energy $E_{\text {int }}^{(\mathrm{ep})}$ in eq. (6). Therefore, partial derivatives of $E_{\text {int }}^{(\mathrm{ep})}$ with respect to the micro-displacements $\mathbf{d}_{\mathrm{m}}$ must be equal to zero. This leads to the internal equilibrium conditions

$$
\mathbf{D}_{\mathrm{mm}} \mathbf{d}_{\mathrm{m}}+\mathbf{D}_{\mathrm{me}} \mathbf{e}-\mathbf{B}_{\mathrm{m}}^{T} \mathbf{D}_{\mathrm{m}} \mathbf{p}_{\mathrm{m}}=\mathbf{0}
$$

where $\mathbf{D}_{\mathrm{mm}}=\mathbf{B}_{\mathrm{m}}^{T} \mathbf{D}_{\mathrm{m}} \mathbf{B}_{\mathrm{m}}$ and $\mathbf{D}_{\mathrm{me}}=\mathbf{B}_{\mathrm{m}}^{T} \mathbf{D}_{\mathrm{m}} \mathbf{B}_{\mathrm{e}}$. The micro-displacements, micro-strains and microstresses can be expressed as

$$
\begin{aligned}
\mathbf{d}_{\mathrm{m}} & =-\mathbf{D}_{\mathrm{mm}}^{-1} \mathbf{D}_{\mathrm{me}} \mathbf{e}+\mathbf{D}_{\mathrm{mm}}^{-1} \mathbf{B}_{\mathrm{m}}^{T} \mathbf{D}_{\mathrm{m}} \mathbf{p}_{\mathrm{m}} \\
\mathbf{e}_{\mathrm{m}} & =\mathbf{B}_{\mathrm{m}} \mathbf{d}_{\mathrm{m}}+\mathbf{B}_{\mathrm{e}} \mathbf{e}=\mathbf{A}_{\mathrm{m}} \mathbf{e}+\mathbf{J}_{\mathrm{m}} \mathbf{p}_{\mathrm{m}} \\
\mathbf{s}_{\mathrm{m}} & =\mathbf{D}_{\mathrm{m}}\left(\mathbf{e}_{\mathrm{m}}-\mathbf{p}_{\mathrm{m}}\right)=\mathbf{D}_{\mathrm{m}} \mathbf{A}_{\mathrm{m}} \mathbf{e}+\mathbf{D}_{\mathrm{m}}\left(\mathbf{J}_{\mathrm{m}}-\mathbf{I}\right) \mathbf{p}_{\mathrm{m}}
\end{aligned}
$$

where $\mathbf{A}_{\mathrm{m}}=\mathbf{B}_{\mathrm{e}}-\mathbf{B}_{\mathrm{m}} \mathbf{D}_{\mathrm{mm}}^{-1} \mathbf{D}_{\mathrm{me}}=\left(\mathbf{I}-\mathbf{J}_{\mathrm{m}}\right) \mathbf{B}_{\mathrm{e}}$ is a certain strain concentration matrix, which transforms the macroscopic strains into elongations of individual links at the micro-level and $\mathbf{J}_{\mathrm{m}}=\mathbf{B}_{\mathrm{m}} \mathbf{D}_{\mathrm{mm}}^{-1} \mathbf{B}_{\mathrm{m}}^{T} \mathbf{D}_{\mathrm{m}}$ is a matrix containing the micro-strains caused by unit plastic strains imposed on individual links, at zero macroscopic strains.

For the periodic cell of volume $V_{\mathrm{p}}$, one can construct the stored energy density $E_{\mathrm{int}}\left(\mathbf{e}, \mathbf{p}_{\mathrm{m}}\right)=\frac{1}{V_{\mathrm{p}}} E_{\text {int }}^{(\mathrm{ep})}\left(\mathbf{A}_{\mathrm{m}} \mathbf{e}+\right.$ $\left.\mathbf{J}_{\mathrm{m}} \mathbf{p}_{\mathrm{m}}, \mathbf{p}_{\mathrm{m}}\right)$ as a function of the macro-strains and the microscopic internal variables, and then eliminate the micro-displacements. The resulting macroscopic stress-strain law can be written as

$$
\mathbf{s}=\mathbf{D e}-\mathbf{D}_{\mathrm{p}} \mathbf{p}_{\mathrm{m}}
$$

where $\mathbf{D}=\mathbf{A}_{\mathrm{m}}^{T} \mathbf{D}_{\mathrm{m}} \mathbf{A}_{\mathrm{m}} / V_{\mathrm{p}}$ is the macroscopic elastic stiffness matrix and $\mathbf{D}_{\mathrm{p}}=\mathbf{A}_{\mathrm{m}}^{T} \mathbf{D}_{\mathrm{m}} / V_{\mathrm{p}}$.

\subsection{Evolution law}

The stress-strain law must be complemented by an evolution law that describes the growth of the plastic internal variables $\mathbf{p}_{\mathrm{m}}$. Such evolution law can be constructed from a dissipation potential. In our simple case, we consider unlimited elastic behavior in compression, and so yielding can occur in tension only. The corresponding loading-unloading conditions can be written as

$$
\dot{\mathbf{p}}_{\mathrm{m}} \geq \mathbf{0}, \quad \mathbf{s}_{\mathrm{m}} \leq \mathbf{s}_{0 \mathrm{~m}}+\mathbf{H}_{\mathrm{m}} \mathbf{p}_{\mathrm{m}}, \quad \dot{\mathbf{p}}_{\mathrm{m}}^{T}\left(\mathbf{s}_{\mathrm{m}}-\mathbf{H}_{\mathrm{m}} \mathbf{p}_{\mathrm{m}}-\mathbf{s}_{0 \mathrm{~m}}\right)=0
$$

where $\mathbf{s}_{0 \mathrm{~m}}$ is the vector containing values of yield stress for all links. 


\subsection{Summary}

The complete description of the model is provided by the following equations and inequalities:

$$
\begin{aligned}
& \mathbf{s}=\mathbf{D e}-\mathbf{D}_{\mathrm{p}} \mathbf{p}_{\mathrm{m}} \\
& \mathbf{s}_{\mathrm{m}}=\mathbf{D}_{\mathrm{m}} \mathbf{A}_{\mathrm{m}} \mathbf{e}+\mathbf{D}_{\mathrm{m}}\left(\mathbf{J}_{\mathrm{m}}-\mathbf{I}\right) \mathbf{p}_{\mathrm{m}} \\
& \dot{\mathbf{p}}_{\mathrm{m}} \geq \mathbf{0}, \quad \mathbf{s}_{\mathrm{m}} \leq \mathbf{s}_{0 \mathrm{~m}}+\mathbf{H}_{\mathrm{m}} \mathbf{p}_{\mathrm{m}}, \quad \dot{\mathbf{p}}_{\mathrm{m}}^{T}\left(\mathbf{s}_{\mathrm{m}}-\mathbf{H}_{\mathrm{m}} \mathbf{p}_{\mathrm{m}}-\mathbf{s}_{0 \mathrm{~m}}\right)=0
\end{aligned}
$$

Here, equation (14) connects the macroscopic stresses, $\mathbf{s}$, with the macroscopic strains, e, and internal variables, $\mathbf{p}_{\mathrm{m}}$. The micro-stresses, $\mathbf{s}_{\mathrm{m}}$, are not independent - they are uniquely linked to $\mathbf{e}$ and $\mathbf{p}_{\mathrm{m}}$ by (15). These micro-stresses are used here only to better understand the meaning of the conditions in (16).

\section{Reduced model}

For large cells, the numerical solution of the proposed problem becomes computationally very demanding due to an extremely high number of internal variables. Therefore, the idea of nonuniform transformation field analysis presented by Michel and Suquet (2003) and extended by Michel and Suquet (2016) is applied in order to reduce the number of internal variables. In this simplification, the vector of microscopic internal variables $\mathbf{p}_{\mathrm{m}}$ is approximated by linear combinations of a few carefully selected fundamental modes of plastic response, referred to as the plastic modes. The approximation can be written as

$$
\mathbf{p}_{\mathrm{m}}=\mathbf{P} \mathbf{p}
$$

where $\mathbf{p}$ is the column vector of plastic mode coefficients that are used in the linear combination and $\mathbf{P}$ is the approximation matrix. Each column of $\mathbf{P}$ represents one fundamental plastic mode.

After applying this approximation, the description of the reduced model can be expressed by the following equations and inequalities:

$$
\begin{gathered}
\mathbf{s}=\mathbf{D e}-\mathbf{D}_{\mathrm{p}} \mathbf{P} \mathbf{p} \\
\mathbf{s}_{\mathrm{m}}=\mathbf{D}_{\mathrm{m}} \mathbf{A}_{\mathrm{m}} \mathbf{e}+\mathbf{D}_{\mathrm{m}}\left(\mathbf{J}_{\mathrm{m}}-\mathbf{I}\right) \mathbf{P} \mathbf{p} \\
\mathbf{P} \dot{\mathbf{p}} \geq \mathbf{0}, \quad \mathbf{P}^{T} \mathbf{s}_{\mathrm{m}} \leq \mathbf{P}^{T} \mathbf{s}_{0 \mathrm{~m}}+\mathbf{P}^{T} \mathbf{H}_{\mathrm{m}} \mathbf{P} \mathbf{p}, \quad \dot{\mathbf{p}}^{T} \mathbf{P}^{T}\left(\mathbf{s}_{\mathrm{m}}-\mathbf{H}_{\mathrm{m}} \mathbf{P} \mathbf{p}-\mathbf{s}_{0 \mathrm{~m}}\right)=0
\end{gathered}
$$

Compared to corresponding formulae in the full model, see eq. (14-16), here the original vector of internal variables (plastic micro-strains) $\mathbf{p}_{\mathrm{m}}$ is replaced by the vector of plastic coefficients $\mathbf{p}$. The length of $\mathbf{p}$ is given by the number of considered plastic modes and therefore is significantly smaller than the length of $\mathbf{p}_{\mathrm{m}}$. This provides a substantial increase of efficiency of the computational procedure.

\section{Results}

The proposed approaches have been implemented in a standard numerical scheme (de Souza Neto et al., 2011). The periodical unit cell with two-dimensional microstructure assembled from $30 \times 30$ initial randomized cells has been submitted to increasing macroscopic strain with a fixed strain ratio $\varepsilon_{x}: \varepsilon_{y}=2: 1$. Such microstructure consists of 1,800 master nodes, 61 ghost nodes, and 5,400 links; see a smaller example in Fig. 1 (a). The hardening modulus is set to $H=0.1 E$ and the yield stress to $s_{0}=0.15 E$ for all links. The corresponding stress-strain diagrams focused in nonlinear areas are plotted in Fig. 1 (b). The exact solution (black) is compared with a reduced simulation with different plastic modes (red and blue).

The plastic modes are precomputed in additional simulations. Each plastic mode corresponds to the distribution of plastic strain $\mathbf{p}_{\mathrm{m}}$ in individual links at a specific loading level. Two different ways of mode selection have been tested. In the first way, the microstructure is loaded separately in $x$ - and $y$-directions. Then individual modes are obtained for each direction with considered loading factors $\left(\varepsilon_{x}\right.$ or $\left.\varepsilon_{y}\right)$ either $\{0.1,0.3,0,5\}$ or $\{0.1,0.2,0.3,0.4,0,5\}$. This procedure leads to 6 (dashed blue line) or 10 (solid blue line) individual modes. In the second case, the microstructure is loaded by imposed strain with a fixed ratio $\varepsilon_{x}: \varepsilon_{y}=2: 1$. And again, loading factors $\left(\varepsilon_{x}\right)$ are considered either as $\{0.1,0.3,0,5\}$ or as $\{0.1,0.2,0.3,0.4,0,5\}$, which leads to 3 (dashed red line) or 5 (solid red line) combined modes. 
The combined modes provide a very accurate approximation. The final stress response is very close to the exact simulation, even if just a few combined modes are used. And a relatively small number of combined modes provides virtually the same result as the exact solution; see the red lines in Fig. 1 (b).

Individual modes have been obtained from different types of simulation. Therefore, they are not able to cover the exact distribution of plastic strain. This leads to a slight overestimation of the final stress response. The error is more significant in the $y$-direction; see the blue lines in Fig. 1 (b). This is caused by the fact that the maximum value of $\varepsilon_{y}$ reached during loading is 0.25 , an thus higher modes $(0.4$ and 0.5$)$ are not activated for this direction. In other words, for the used setting, the $x$-direction is approximated with more modes than the $y$-direction.

As one can expect, employment of more modes provide a smoother stress response, because particular modes can be activated continuously during the loading process.

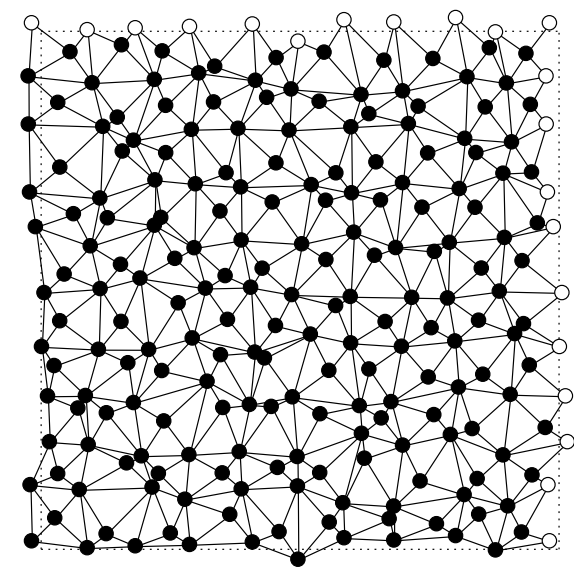

(a)

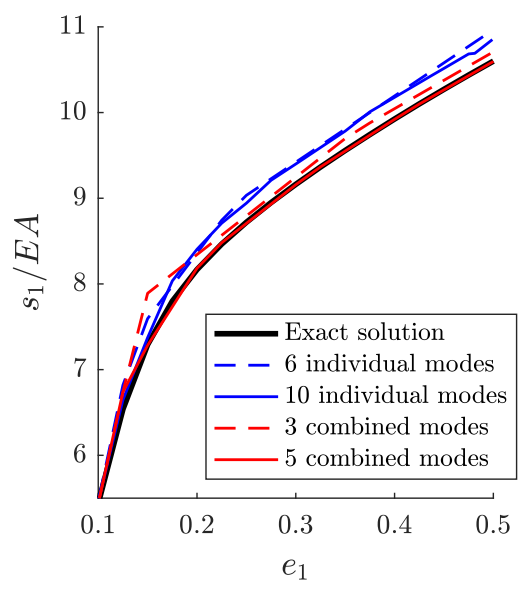

(b)

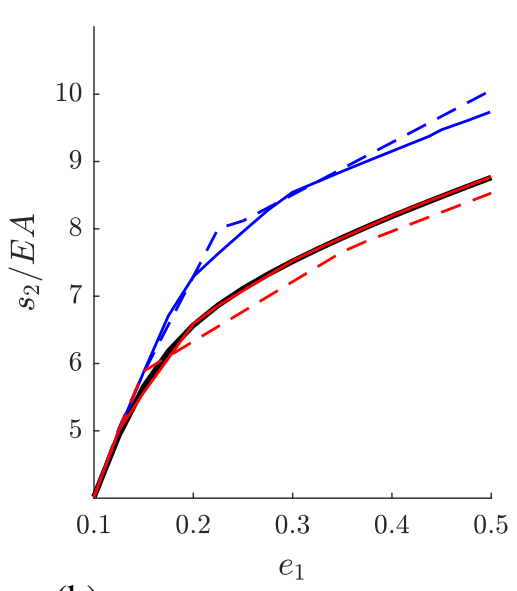

)

Fig. 1: (a) Example of randomized microstructure with $10 \times 10$ cells and periodic boundaries (dotted line), master particles (filled) and ghost particles (hollow); (b) nonlinear parts of stress-strain diagrams for microstructure with $30 \times 30$ initial cells loaded by macroscopic strain with strain ratio $\varepsilon_{x}: \varepsilon_{y}=2: 1$.

\section{Conclusions}

The presented results have shown that model reduction based on the nonuniform transformation field analysis can be used to simplify the given problem and significantly reduce the number of internal variables while the error in the macroscopic results remains acceptable.

The selection of plastic modes has turned out to be essential for the accuracy of the presented simplification. Therefore, future research will focus on the development of appropriate techniques of generating universal plastic modes.

\section{Acknowledgments}

Financial support of this research received from the Grant Agency of the Czech Technical University in Prague SGS19/032/OHK1/1T/11 and from the Czech Science Foundation (GAČR 17-04150J) is gratefully acknowledged.

\section{References}

Michel, J-C. and Suquet, P. (2003) Nonuniform transformation field analysis. International journal of solids and structures, 40, 25, pp 6937-6955.

Michel, J-C. and Suquet, P. (2016) A model-reduction approach in micromechanics of materials preserving the variational structure of constitutive relations. Journal of the Mechanics and Physics of Solids, 90, pp 254-285.

de Souza Neto, E.A., Peric, D. and Owen, D.R.J. (2011) Computational methods for plasticity: theory and applications. John Wiley \& Sons. 